\title{
Interpretation and Diplomacy Aspects of Authority and Care in Imaging Reports
}

\author{
Werner A. Golder ${ }^{1}$
}

Published online: 14 August 2017

(C) Society for Imaging Informatics in Medicine 2017

\begin{abstract}
Whereas the creativity and intellectual power of the radiologist are measured against his/her written report, the value of the message will not only be judged by the precision of the medical statement. The same result can be attributed to different words. Numerous common and accidental factors exert influence on the decision on what is said and what is not said, how it is assessed and what is ignored. The less certain a diagnosis is and the less favourable its possible consequences are, the more subtleties and periphrases are to be expected within the report. The decision on the nature and the volume of the written report will not only be taken by the time of recording, but is likewise prepared by the knowledge of the patient's history and symptoms, the personal relationship to $\mathrm{him} /$ her as well as by a set of conditions throughout the inspection of the images. The intuition that accompanies the information transfer in imaging diagnostics does not only explain the differences in volume and depth of diagnosis and differential diagnosis, but also the range of diagnostic and therapeutic recommendations.
\end{abstract}

Keywords X-ray report · Interpretation · Objectivity · Recommendation $\cdot$ Empathy

Key Points - The clinician has a flair for different ways of expressing. - Numerous readers take note of even subtle distinctions of the choice of words.

- The reaction of the recipient is influenced by the terminology.

- Radiologists translate their observations in a personal manner.

- The diagnostic message is more than a declaratory decree.

Werner A. Golder

werner.golder@orange.fr

1 Association d'Imagerie Médicale, Avignon, 23, rue de l'Oriflamme, 84000 Avignon, France
The analytical performance aligned with the records of diagnostic imaging is often referred to as interpretation [1]. However, this term describes only incompletely the enquiry that comes about by stages. It suggests to the reader that the radiologist reads a finished product like the philologist a poem or the musician a score. But the radiograph is an object, the production and quality of which is essentially influenced and the properties of which can be modified by the analyst. In addition, the examiner is determined by a series of conditions, that are more or less foreseeable, that are perceived more or less intentionally and that unfold more or less strong effects. What will finally be expressed in the record and in which words the message will be clothed is initiated in the approaches and the surroundings of the study. Thus, the authority of the radiologist on the choice of words is challenged in each particular case one more time. The comparative reading of medical imaging reports shows, to what extent the willingness and the ability to take notice of the various challenges differ $[2,3]$.

The broad range of the information transfer has two fundamental consequences. Firstly, it exercises an influence on the response and reaction of the recipient to the presented result. Regardless of whether the diagnostic statement is correct or only more or less appropriate, the feedback that it evokes with the questioner is shaped by the choice of words. Secondly, it controls the degree of satisfaction of the recipient with the diagnostic performance and his readiness to continue the cooperation with the radiologist on the same or another scale. Both effects are more pronounced in outpatient than in inpatient imaging diagnostics. Therefore, a change between the two modi operandi will unveil these effects especially well, indeed in both directions.

The report of the radiologist is never totally objective. Striving for an unprejudiced statement will be limited on a mere basis of the formal conditions in the running-up to the 
examination. The very fact that a certain imaging procedure has been asked by the referring colleague conditions the radiologist and triggers corresponding expectations with him. Each report is the individual answer to a new diagnostic challenge and more than the description and assessment of contours and contrasts. Each text has a personal character, may it also be more or less well hidden beneath the facts. Even the routine use of prefabricated text elements or a structured protocol does not banish the free choice of words by the radiologist to silence.

\section{Expectations Shape the Report}

Even before the radiologist sets about the examination and takes a look at the images, respectively, the diagnostic information is already shaped by the way the request has been submitted by the referring physician. The coordination of the formal information (age, sex, profession of the patient, discipline of the referring doctor) with epidemiologic knowledge and clinical skill produces certain anticipations. Accordingly, the radiologist adjusts his horizon of expectations. The careful inspection of the common data permits to rule out a number of diagnoses and differential diagnoses to a large extent, but promotes others conversely. A similar preliminary decision arises from the information, if it refers to a first or a consecutive/ repetitive examination, a screening examination or an examination performed within the bounds of a clinical study, respectively. When there is a lack of that kind of details, the report is threatened by a loss of accuracy, as the radiologist cannot reply to one or more particular clinical questions in a selective manner $[4,5]$. Accordingly, a diagnostic imaging report that is prepared without sufficient knowledge of the underlying medical history tends to be both longer and more diffuse than its counterparts and appears to suffer from a lack of rigour and therefore to be less reliable. Almost inevitably, within such records, one or more elements are described and assessed that are not coupled with the current clinical problem. Nevertheless, this shortcoming does not reduce their value fundamentally. By the same token, the examiner is more prepared to come to a definitive decision, if he/she performs an imaging modality that is inevitably connected with a close relationship between him/her and the patient, e.g. an ultrasound examination. Consequently, an objectively minor finding will be assessed even as affirmative when it is in favour with the clinical context.

By general consent, precise clinical data and specific questions of the referring doctor are the best guide to conclusive and therapeutically valuable statements of the radiologist. On the other side, just this information puts pressure on the interpreter at least to a certain degree. The referring colleague expects the radiologist to solve the problem presented both with an appropriate methodical approach and intellectual excellence and to put the result into an equally clever and finely tuned report. If the radiologist does not succeed in carrying out the order in a satisfying manner as far as the contents are concerned, the choice of words for the report runs into a problem. It is less demanding to confirm a suspected diagnosis than to reject it and to present an alternative solution that may be less obvious in the light of the medical history and the clinical findings. Thus, in order to show consideration for the referring doctor, the radiologist can offer a diagnosis with the ring of conviction as well as without emphasis. Quite rarely, he/she will be criticized or even charged with a suspected diagnosis turning out to be erroneous. Likewise, the knowledge of the medical history may only infrequently steer the radiologist away from a correct diagnosis. Even so a careful admonition is generally preferred to a shrill alert. A similar challenge to the choice of words can be recognized in the part the radiologist has to play within the framework of post-interventional control examinations as supervisor of the effects of the measures that have been carried out by the colleagues of other disciplines. Whereas a successful outcome can be adequately reported in a few proper words, the depiction of the opposite may be clothed into playful arguments or half-open statements. All radiologists who are not only engaged in the diagnostic, but also in the interventional sector, can learn from the written criticism of his/her own efforts by their colleagues, how other disciplines deal with these difficulties.

Many doctors prefer to integrate the radiological diagnosis in the clinical context and to present it to the patient in person. All the same, this rule is only respected in hospitals. Within his/her private office, the radiologist is accustomed to present the images and findings to the patient and to talk about the possible consequences in order to alleviate his/her anxiety immediately afterwards. In some countries, that is also his/ her due. Subsequent to the reading of the final report, some patients return into the radiologist's practice in order to be more thoroughly informed about details in the light of complementary reports or with respect to planned therapeutic measures. Also and just in this situation, the doctor will benefit from an evaluation that does not contain ambiguous statements or expressions. That holds especially true for tentative diagnoses or borderline findings. The radiologist does a favour both to himself and to the patient, if he exercises restraint in doubtful cases and if he talks about the frontiers of his statements unequivocally. Differentials that include more than three or four diagnostic choices are inappropriate both inside and outside academic institutions. They are not only considered as book knowledge, but mostly they are so. Likewise, it is a sign of superiority, if unsuspicious findings and commonplace statements are presented as briefly as possible. Regardless of the type of the imaging procedure applied, a term like "otherwise unremarkable" can serve as an abbreviation, that is in every respect adequate for all normal or negative 
findings. The intelligent radiologist will avoid utterances like "clinical correlation is suggested" as a matter of principle, since they can be easily misunderstood as a substitute for clear diagnostic conclusions.

Occasionally, the performance of diagnostic imaging and imaging-guided procedures is hampered or their quality is adversely affected by the patient. Whether he/she has overweight or is bloated or cannot stop breathing for a sufficient period or does not keep quiet in other respects, there is a long list of factors and situations related to the patient and the underlying disease that might reduce the quality of the radiographs. The examiner is both bound and allowed to call such an obstacle to the reader's attention in the technical section of the report. All the same, he will choose his/her words so deliberately that neither the wording is disrespectful nor the patient even allusively accused to be fully or partially responsible for the shortages of the examination.

\section{Recommendations, Not Rectifications}

From the very beginning, incidental findings have been a frequent phenomenon in diagnostic radiology. Their increase in number has been brought about by the fact that often, for instance as part of computed and magnetic resonance tomography (keyword: whole body imaging), more than one region is depicted. In a similar manner, screening procedures foster the discovery of unexpected findings. Largely, the examiner becomes aware of innocent anomalies whose description and assessment can be settled in an epilogue. However, incidental findings of unknown nature and type or other results that demand action without delay or that will give rise to other dramatic consequences can seriously influence the radiologist's choice of words and tone. In order to prevent secondary damages and/or to keep them to a minimum, the record is immediately conveyed both to the patient and the referring physician in private or by telephone. These precautionary arrangements are capable to restrain the surprise effect that is inevitably connected with the exclusively written transfer of an appalling diagnosis. Subsequently, these preparatory instructions are recorded in the final report.

Both laconic brevity and baroque love of details can bring $\mathrm{X}$-ray reports into discredit. In either case, the reader will not be convinced that he is taken seriously. Didactic comments (notorious example: "Of course a meniscus lesion cannot be ruled out in view of the normal X-ray findings of the knee") should be avoided without exception and irrespective of the specialization of the recipient. But even standard expressions (for instance "as far as evident from the today's examination") can lead to considerable annoyance and a corresponding loss of confidence and dissatisfaction, notably when the reader will discover them more than once in a while.
The diagnostic radiologist is not a merchant, but an authorized agent. However, he/she has to sell the information he/she has been asked for. The trust that has been transferred to him/her with the referral is answered by a combination of attention, perception and discernment within the report. A clearly articulated verbal association of X-ray findings and imaging characteristics with the clinical diagnosis (as evident from a formula like "... as a sign for...") is highly esteemed by the majority of the readers. They consider this way of assessment as a symbol of clinical competency and the effort for a causally and practiceorientated interpretation. By the same token, if the descriptive elements dominate and the author is obviously not willing to commit himself to a diagnosis, this policy is interpreted as a proof that he/she is reluctant to take responsibility and prefers to leave the final interpretation of the images to the bedside assessment [6-8].

Many X-ray reports come to a close with one or more diagnostic and/or therapeutic recommendations, usually for additional investigations or patient referral. Advices like those have to be harmonized with the clinical situation in style and tone. This demand holds true even for such a frequently articulated recommendation like the comparison with former examinations and prior reports. Even if the demand for a followup is always justified, the examiner might be misunderstood in his/her manner of handling and blamed for inertia and unjustified assignment of a genuine radiologic task. In that case, it seems to be outstandingly clever to offer the own commitment in pursuit of supplementary imaging material at least as an alternative. By comparison, if recommendations are expressed for controls and subsequent measures in the field of diagnostic imaging, the radiologist should avoid referring to the institution where he/she works. In assuming this attitude, he/she steers clear of pretending subsequent imaging measures or even making an appointment and as a consequence demanding part of the decision-making power of the clinician. If further non-radiologic diagnostic efforts are recommended, only the discipline is referred to, not a single or particular procedure. That kind of discretion is demanded by the respect for the skill and competency of the addressed colleagues. Finally, radiologic interventions with therapeutic intention are offered on the understanding that a respective agreement has been achieved in the course of a personal consultation or a conference between radiologists and clinicians.

\section{References}

1. Larson DB, Duncan JR, Nagy PG, Kruskal JB: Guide to effective quality improvement reporting in radiology. Radiology 271(2):561573, 2014

2. Aideyan UO, Berbaum K, Smith WI: Influence of prior radiologic information on the interpretation of radiologic examinations. Acad Radiol. 2(3):205-208, 1995 
3. Elmore GJ, Wells CK, Howard DH, Feinstein AR: The impact of clinical history on mammographic interpretations. J Am Med Assoc. 227(1):49-52, 1997

4. Leclère $\mathrm{J}$, Leclère $\mathrm{C}$, Ollivier L: Le compte-rendu radiologique: à fond la forme. J Radiol. 88(2):297-303, 2007

5. Mortani Barbosa, Jr EJ, Lynch MC, Langlotz CP, Gefter WB: Optimization of radiology reports for intensive care unit portable chest radiographs: perceptions and preferences of radiologists and
ICU practitioners. J Thorac Imaging 31(1):43-48, 2016 Jan. doi: 10.1097/RTI.0000000000000165

6. Naik SS, Hanbridge A: Wilson SR radiology reports: examining radiologist and clinician preferences regarding style and content. Amer J Roentgenol 176(3):591-598, 2001

7. Hegedüs V: Which way? Europ J Radiol 9:1-2, 1989

8. Göthlin JH: Teaching medical students diagnostic radiology. Europ J Radiol 9:3, 1989 